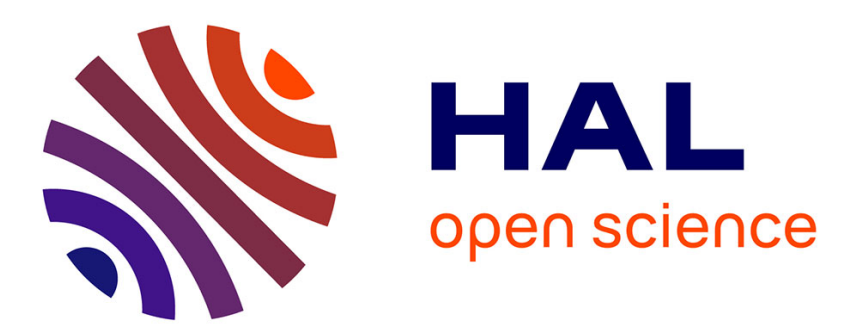

\title{
A sequential test for autonomous localisation of map errors for driving assistance systems
}

\author{
Clément Zinoune, Philippe Bonnifait, Javier Ibañez-Guzmán
}

\section{To cite this version:}

Clément Zinoune, Philippe Bonnifait, Javier Ibañez-Guzmán. A sequential test for autonomous localisation of map errors for driving assistance systems. IEEE Transactions on Intelligent Transportation Systems, 2012, pp.1377-1382. hal-00841573

\section{HAL Id: hal-00841573 \\ https://hal.science/hal-00841573}

Submitted on 5 Jul 2013

HAL is a multi-disciplinary open access archive for the deposit and dissemination of scientific research documents, whether they are published or not. The documents may come from teaching and research institutions in France or abroad, or from public or private research centers.
L'archive ouverte pluridisciplinaire HAL, est destinée au dépôt et à la diffusion de documents scientifiques de niveau recherche, publiés ou non, émanant des établissements d'enseignement et de recherche français ou étrangers, des laboratoires publics ou privés. 


\title{
A sequential test for autonomous localisation of map errors for driving assistance systems
}

\author{
Clément Zinoune ${ }^{1,2}$, Philippe Bonnifait ${ }^{1}$, Javier Ibañez-Guzmán ${ }^{2}$
}

\begin{abstract}
Driving assistance systems are progressively introduced to enhance safety and comfort in passenger vehicles. They increasingly rely on information stored in digital navigation maps. However maps can be obsolete or contain errors, resulting in malfunctions of context based driving assistance systems and possibly generating hazardous situations. This paper aims at making the vehicle able to detect and localise map errors in an autonomous manner using its embedded sensors. The proposed approach relies on the sequential generation and monitoring of residuals. The vehicle estimated trajectory is compared statistically with the geometric data in the map. The approach allows driving assistance functions to know if they can rely on the map in real-time and to store this information for future journeys. The method is very efficient in terms of computational load which makes embedded applications possible. Performance is assessed using vehicle data acquired in real traffic conditions, which is then compared with an outdated navigation map.
\end{abstract}

\section{INTRODUCTION}

Driving assisting functions are becoming standard in new vehicles. Organizations such as the European New Car Safety Programme (Euro NCAP) [1], which provide a classification in terms of safety to new vehicles, are incorporating the use of Advanced Driving Assistance Systems (ADAS) into their criteria. Contextual information about the area where the vehicle evolves is essential for such systems. The presence of obstacles in the vehicle trajectory, the forthcoming road curvature and the current speed limit may be detected by the vehicle on-board exteroceptive sensors like laser scanners, radars or cameras. Whilst these have the potential to provide a rich understanding of the vehicle situation, their deployment in all types of traffic conditions is limited not only by the physics associated to them (e.g. field of view), but also by their cost. To enhance their performance, they are often combined with contextual data extracted from stored maps.

Digital maps are becoming the central component of some ADAS and of driverless passenger vehicles [2], [3]. However, they inevitably hold errors due to the constant evolution of road networks and errors during the mapping process. These may cause severe malfunctions of map-aided ADAS. It is therefore crucial for systems to know whether the stored digital maps can be relied upon. The quality of a given map may be evaluated by comparing them with reference maps or aerial images. however this requires offline

\footnotetext{
1 Heudiasyc UMR CNRS 7253, Université de Technologie de Compiègne, France.philippe.bonnifait@hds.utc.fr, clement.zinoune@utc.fr

2 Renault S.A.S, France. javier.ibanez-guzman@ renault.com
}

and centralised processing, with results being available to vehicles at considerable delays.

This paper presents a sequential approach aiming at making the vehicle capable to assess autonomously the on-board digital map used for ADAS systems whilst in motion. The vehicle position is first estimated using an Extended Kalman Filter (EKF) based on Global Navigation Satellite System (GNSS) and vehicle embedded sensors. This estimation is then compared with the vehicle's map-matched position provided by the navigation system. It is shown that the detection of an error in the digital map is equivalent to ascertain a change in the mean of a random variable. This paper focuses on the reduction of the time required for the detection of the beginning and the end of the map errors. Moreover, a precise localisation of map errors is done and stored in the vehicle's memory for future journeys. The system performance is evaluated using data acquired in real traffic conditions and compared with empirical mean and Neyman Pearson tests. This is the main contribution of the paper.

The paper is organized as follows: Section II presents a review of existing methods and formulates the problem. The manner in which the vehicle trajectory and positioning is estimated using embedded vehicle data is presented in Section III. The proposed approach for map error detection is described in Section IV. Results obtained from tests with real data are presented and statistically analysed in Section V. Section VI concludes the paper.

\section{State of The ART AND PROBlem STATEMENT}

Contributions to the maps assessment exist within the geographic information science (geomatics) domain [4]. The basis of these methods resides in comparing two maps using metrics which can be as various as the elements to assess. Vector maps are made of three basic entities, vertices, lines and surfaces. Only the two firsts are relevant for our application since surfaces are not used by ADAS. The simplest way to measure the similarity of two points of the space is the Euclidean distance. which is defined for two points $A=\left(x_{A}, y_{A}\right)$ and $B=\left(x_{B}, y_{B}\right)$ by $d_{E}$ in Eq. (1).

$$
d_{E}(A, B)=\sqrt{\left(x_{B}-x_{A}\right)^{2}+\left(y_{B}-y_{A}\right)^{2}}
$$

The similarity between two curves may be measured as the maximum gap between them using the Hausdorff distance [5]. This is a good metric to detect road offsets and some dissimilarities in the curves shapes. However it may be 


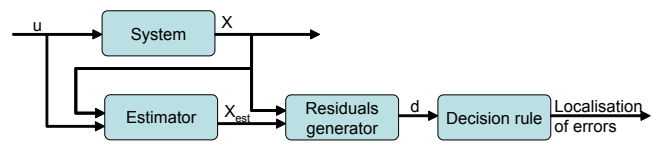

Fig. 1. Typical block diagram of system monitoring

tricked if the segments have different lengths or very different shapes [6]. The mean distance introduced in [7] takes into account the surface between the curves.

In general, each metric allows highlighting a precise characteristic of similarity between geographical features (distance, shape, likelihood, ....). It is then suitable to adopt a fault detection strategy to interpret their behaviour.

A very wide range of mathematical and statistical methods have been used to compare sets of geographical data. In [8], the authors use fuzzy logic to compute an outlier index that expresses how a geographical object belongs to its spatiotemporal neighbourhood. This approach aims at detecting errors as well as temporal changes in maps.

Map creation and assessment may be done straight from aerial imagery [9], [10], [11]. The roads are extracted thanks to image processing methods and compared to an existing digital map.

The vehicles themselves can be used to create or to refine maps. In [12], [13], a large number of GNSS traces are used to infer the road map of an area or to increase the map precision with probabilistic clustering techniques.

In [14], the authors adapt probabilistic simultaneous localisation and mapping (SLAM) methods to increase the map's level of precision. The roads are modelled by cubic splines and used as state variables in the positioning filter. The parameters of the splines are updated while the robot evolves in its environment.

Map comparison requires that the two maps to be compared are accessible. These may come from geospatial databases or from aerial images. Also, the execution time is not a critical issue while the algorithms are run offline. However, in automotive applications the constraints are different. Vehicle's system architectures make difficult to have access to the digital navigation map as a whole. Only information about the road environment close to the vehicle is available. Moreover, embedded algorithms must be efficient enough to be run online by inexpensive ECUs. Therefore approaches proposed in system diagnosis domain are then very relevant in our problem.

Redundant sources of information may be used to detect faults in a system using statistical methods [15]. In [16] cumulative sum is used to detect abrupt changes of the Kalman filter covariance matrix and thus isolate faults in a robot's positioning system.

System monitoring approaches are very promising for intelligent vehicles applications as they require reasonably low computational capabilities. The system architecture adopted in this work detailed in Fig. 1. The state of the system to monitor is estimated using its available inputs and outputs. Residuals are then generated and allow error detection and localisation thanks to a set of statistical rules.

\section{VEHICLE POSITIONING}

The real-time vehicle positioning is done by a loosely coupled Extended Kalman Filter (EKF). It uses GNSS fixes when available to correct the proprioceptive estimation [17]. The state representation is shown in Eq. (2).

$$
\begin{aligned}
& \left\{\begin{array}{c}
X_{k}=f\left(X_{k-1}, U_{k}\right)+\alpha_{k} \\
Y_{k}=H \cdot X_{k}+\beta_{k}
\end{array}\right.
\end{aligned}
$$

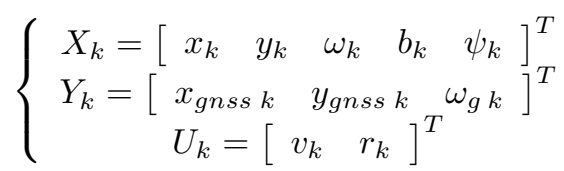

The state, observation and input vectors $X, Y$ and $U$ respectively, are detailed in Eq. (3), where index $k$ denotes the $k^{t h}$ time step. In the state vector, $\left(\begin{array}{ll}x & y\end{array}\right)$ are the vehicle's coordinates in a East-North reference frame. $\psi$ and $\omega$ stand for the vehicle's heading (clockwise from the north direction) and yaw rate (rotation rate around the $U p$ axis) respectively. Finally, $b$ is the vehicle's gyroscope bias as defined in Eq. (5). The observation vector contains the vehicle's position according to the GNSS observation ( $\left.\begin{array}{ll}x_{\text {gnss }} & y_{\text {gnss }}\end{array}\right)$ and yaw rate measured by the vehicle's gyroscope $\omega_{g}$. The input vector is composed of the vehicle's speed $v$ and the speed difference between the rear vehicle's wheels $r$.

The model and observation noises are $\alpha$ and $\beta$ respectively, and supposed to be white and zero mean.

The state transition function $f$ depends on the input $U$ and on the state itself. It is defined in Eq. (4) where $T$ is the sampling period and $l$ is the vehicle's rear track (the distance between the two rear wheels). The observation model $H$ used here is given in Eq. (7).

$$
\begin{gathered}
f\left(X_{k-1}, U_{k}\right)=\left[\begin{array}{c}
x_{k}+T \cdot v_{k} \cdot \sin \left(\psi_{k}\right) \\
y_{k}+T \cdot v_{k} \cdot \cos \left(\psi_{k}\right) \\
l \cdot r_{k} / 2 \\
b_{k} \\
\psi_{k}-T \cdot \omega_{k}
\end{array}\right] \\
\omega_{g}=\omega+b \\
r=l \cdot \omega \\
H=\left[\begin{array}{lllll}
1 & 0 & 0 & 0 & 0 \\
0 & 1 & 0 & 0 & 0 \\
0 & 0 & 1 & 1 & 0
\end{array}\right]
\end{gathered}
$$

\section{MAP ERROR DETECTION AND LOCALISATION}

Statistical tests are appropriate to evaluate parameters of a probability law based on set of outcomes. In our application, we aim at detecting a change of the mean of the probability density function (PDF) of a set of observed data while the standard deviation of this PDF is in the same order of magnitude than the expected mean gap. Page's trend test works sequentially and is especially efficient for stream data. 
In the following sections, the test principles are recalled in general terms and then applied to autonomous map error detection.

\section{A. Page's trend test}

Page's test consists in statistically detecting a change in the mean of a random variable [15]. Let us consider $N$ samples $d_{i}$ of a random variable $D$. The likelihood of two hypotheses $H_{0}$ and $H_{1}$ are compared. The first one states that $D$ has a constant mean $\mu_{0}$ among the $N$ samples. The second one assumes that the mean of $D$ was $\mu_{0}$ for the first $r-1$ samples and $\mu_{1}$ for samples $r$ to $N$ :

$$
\begin{gathered}
H_{0}: d_{i}=\mu_{0}+b_{i}, i=1, \ldots, N \\
H_{1}:\left\{\begin{array}{c}
d_{i}=\mu_{0}+b_{i}, i=1, \ldots, r-1 \\
d_{i}=\mu_{1}+b_{i}, i=r, \ldots, N
\end{array}\right.
\end{gathered}
$$

where $b$ is a zero-mean noise of standard deviation $\sigma$. The generalized likelihood ratio of both hypotheses is given by Eq. (9).

$$
\Lambda(D)=\frac{\prod_{i=1}^{N} p\left(d_{i}, r \mid H_{1}\right)}{\prod_{i=1}^{N} p\left(d_{i} \mid H_{0}\right)}
$$

Since the likelihood of the alternative hypothesis $H_{1}$ depends of an unknown parameter $r$, its maximum likelihood estimation is considered.

$$
\Lambda(D)=\frac{\sup _{r}\left(\prod_{i=1}^{r-1} p\left(d_{i} \mid H_{1}\right) \prod_{i=r}^{N} p\left(d_{i} \mid H_{1}\right)\right)}{\prod_{i=1}^{N} p\left(d_{i} \mid H_{0}\right)}
$$

As the likelihood of the null hypothesis $H_{0}$ does not depend on $r$ and having $\prod_{i=1}^{r-1} p\left(d_{i} \mid H_{1}\right)=\prod_{i=1}^{r-1} p\left(d_{i} \mid H_{0}\right)$, the likelihood ratio can be simplified as follows:

$$
\Lambda(D)=\sup _{r}\left(\prod_{i=r}^{N} \frac{p\left(d_{i} \mid H_{1}\right)}{p\left(d_{i} \mid H_{0}\right)}\right)
$$

Let $\delta$ denote the mean gap $\left(\delta=\mu_{1}-\mu_{0}\right)$. Under Gaussian assumption, one can get [18]:

$$
\ln (\Lambda(D))=\frac{\delta}{\sigma^{2}} \sup _{r}\left(\sum_{i=r}^{N}\left(d_{i}-\mu_{0}-\frac{\delta}{2}\right)\right)
$$

The decision of choosing either $H_{0}$ or $H_{1}$ is made by comparing the likelihood ratio with a threshold $\lambda$ :

$$
\left\{\begin{array}{l}
H_{0}: \ln (\Lambda(D))<\ln (\lambda) \\
H_{1}: \ln (\Lambda(D))>\ln (\lambda)
\end{array}\right.
$$

For real time implementation, it is especially convenient to formulate the test sequentially. Let us then define the cumulative sum as in Eq. (14).

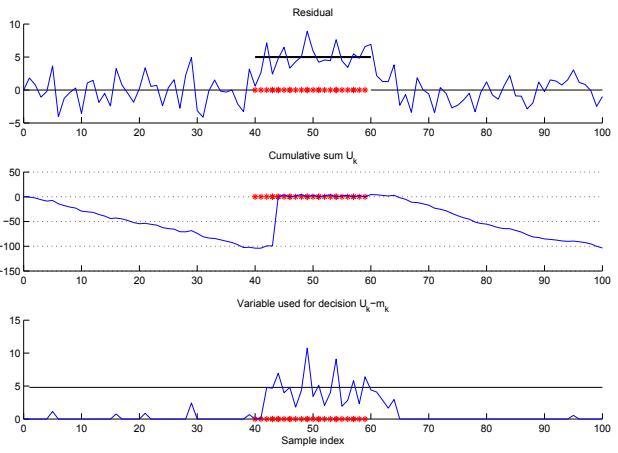

Fig. 2. Illustrative example of Page's test. The signal to consider is plotted on the top graph. The corresponding cumulative sum and decision variable are beyond. The red stars denote the sample indexes for wich the test has detected a mean change.

$$
S_{r}^{N}\left(\mu_{0}, \delta\right)=\delta \sum_{i=r}^{N}\left(d_{i}-\mu_{0}-\frac{\delta}{2}\right)
$$

which can be re-written:

$$
S_{1}^{N}\left(\mu_{0}, \delta\right)=S_{1}^{r-1}\left(\mu_{0}, \delta\right)+S_{r}^{N}\left(\mu_{0}, \delta\right)
$$

The decision rule then becomes:

$$
\left\{\begin{array}{l}
H_{0}: S_{1}^{N}\left(\mu_{0}, \delta\right)-\inf _{r}\left(S_{1}^{r-1}\left(\mu_{0}, \delta\right)\right)<\gamma \\
H_{1}: S_{1}^{N}\left(\mu_{0}, \delta\right)-\inf _{r}\left(S_{1}^{r-1}\left(\mu_{0}, \delta\right)\right)>\gamma
\end{array}\right.
$$

where $\gamma=\sigma^{2} \ln (\lambda)$.

Let $\delta_{m}$ be the minimum value of $\delta$ which must be detected. The test is split into two sub-tests running in parallel, the first aiming at detecting a mean growth and the other a decrease of the mean.

Finally, at the current time $k$, a mean growth is detected as soon as Eq. (17) is true.

$$
U_{k}-m_{k}>\gamma
$$

where

$$
\begin{gathered}
U_{k}=U_{k-1}+d_{k}-\mu_{0}-\frac{\delta}{2} \\
m_{k}=\min \left(m_{k-1}, U_{k}\right)
\end{gathered}
$$

Conversely, a mean decrease is detected when:

$$
M_{k}-T_{k}<\gamma
$$

where

$$
\begin{gathered}
T_{k}=T_{k-1}+d_{k}-\mu_{0}+\frac{\delta}{2} \\
M_{k}=\max \left(M_{k-1}, T_{k}\right)
\end{gathered}
$$

As soon as threshold $\gamma$ is reached, the cumulative sums are reset to zero. The actual mean change happened at the last time $m$ (resp. $M$ ) has reached its minimum (resp. maximum) before crossing $\gamma$. This test is then very efficient in terms of required computational load. Indeed, at every new time step, it only requires to make additions and comparisons of scalar variables. 
The choice of $\gamma$ has consequences on the false alarm probability. It is not possible to express it formally because the probability density function of hypothesis $H_{1}$ depends of an unknown parameter $r$. However, it can be set based on the number $h$ of estimated parameters in the probability density function (PDF) and on the number $n$ of standard deviations: $\gamma=2 \cdot h \cdot n \cdot \sigma / \delta_{m}$. Here the mean is the only estimated parameter of the PDF and $n$ is set to 2 as nominal tuning which is a good compromise between false alarm rate and time to detection [18]. Then $\gamma=4 \cdot \sigma / \delta_{m}$

Let us consider the example shown in Fig. 2. The residual presents a mean jump between indexes 40 and 60. The decision variable crosses the threshold at index 44. Thanks to the cumulative sum it is possible to find the origin of the fault back at index 40 .

\section{B. Residuals generation for map errors}

This section develops the residuals generator as indicated in Fig. 1. The residuals are computed between map-matched points and estimated vehicle positions provided by the estimator bloc (EKF). The residuals that we use are the lateral and longitudinal offsets in a frame $\mathcal{R}_{1}$ aligned with the road.

Let us consider map-matching as a random process that provides an estimation $M$ of the true vehicle position $X$ in the axis system $\mathcal{R}_{1}$ :

$$
M=X+\alpha
$$

where $\alpha$ is a zero-mean noise with a diagonal covariance matrix with $\sigma_{a}^{2}$ and $\sigma_{b}^{2}$ as components (longitudinal and lateral standard deviations of the map matched position along the road segment).

The components used from the state vector are denoted by $X_{\text {est }}=(x, y)^{T}$ in the East-North plane $\mathcal{R}_{0}$ locally tangent to Earth with the covariance matrix $P$ of the estimation error $\beta$ :

$$
P=\left[\begin{array}{cc}
\sigma_{x}^{2} & \sigma_{x y}^{2} \\
\sigma_{x y}^{2} & \sigma_{y}^{2}
\end{array}\right]_{\mathcal{R}_{0}}
$$

In order to make the residuals independent on the road direction, an isotropic approach consists in using the outer circle of the ellipsoid. Its radius is $\lambda=\max \left(\lambda_{i}\right), \lambda_{i}$ being the eigenvalues of $P$. So the covariance matrix expressed in $\mathcal{R}_{1}$ is $\lambda \cdot I$ (with $I$ being the identity matrix).

In $\mathcal{R}_{1}$, the difference between the map matched and estimated positions is given by $L$ which has two independent components.

$$
L=\left[\begin{array}{l}
d \\
e
\end{array}\right]=M-X_{e s t}=\alpha-\beta
$$

Under the hypothesis of independent errors, the lateral residual $d$ and the longitudinal residual $e$ have the following variances.

$$
\begin{aligned}
& \sigma_{d}^{2}=\lambda+\sigma_{b}^{2} \\
& \sigma_{e}^{2}=\lambda+\sigma_{a}^{2}
\end{aligned}
$$

The detection of geometrical errors in maps can now be made by running the Page's tests simultaneously on two independent residuals: $d$ and $e$. The means employed in hypotheses $H_{0}$ are set to zero since an error-free road link is, by definition, centred on its true location. Oppositely, the means used in hypotheses $H_{1}$ are set to the maximum acceptable bias in the map.

\section{EXPERIMENTAL RESULTS}

In this section, the experimental data gathered in real traffic conditions and used for method validation is first detailed. The method developed in the previous section is then assessed and compared to two standard methods based on fixed length sliding windows. The algorithms are tested on four real areas chosen to be representative of critical map errors.

The method was tested in real conditions thanks to a Renault Espace standard passenger vehicle. The localisation system on board was a standard single frequency GNSS receiver. A high grade inertial navigation system (INS) [19] tightly coupled to a dual frequency GNSS receiver was used as position ground truth.

Via the CAN-bus, we recorded the vehicle's odometer, speed, rear wheel speed difference and yaw rate as required by the vehicle positioning system developed in section III.

An out-of-date commercial digital map designed for advanced navigation purposes was used. This presents the advantage of containing roads that have been modified since the creation of the map as well as roads that have not changed. This is very convenient for testing an error detection system in real conditions. The map-matched points provided by the navigation engine were broadcast on the CAN-bus.

\section{A. Analysis methodology}

We aim at evaluating the performance of a fault monitoring system in terms of distance to alert, distance to recovery (an adaptation of time-to-alert and time-to-recovery) and accuracy of map error localisation. In this section, we present the metrics used for this evaluation.

As shown in Fig. 3, the distance to alert $\delta_{H_{0} \rightarrow H_{1}}$ is the distance travelled by the vehicle before detecting an error. Reciprocally, $\delta_{H_{1} \rightarrow H_{0}}$ is the recovery distance. As soon as the vehicle passed through the map error, the fault is localised and stored in the map for future use. $e_{H_{1} \mid H_{0}}$ denotes the length of the road that has been identified as erroneous while being actually fault free. Reciprocally, $e_{H_{0} \mid H_{1}}$ stands for the length of road that actually contains an error that has not been detected by the algorithm.

The ground truth trajectory has been employed to localise manually the map errors using a step-by-step data player.

\section{B. Other tests used for comparison}

The performance of Page's trend test has been compared to two other classical methods based on fixed length sliding window. These both aim to discriminate between $H_{0}$ and $H_{1}$ : 


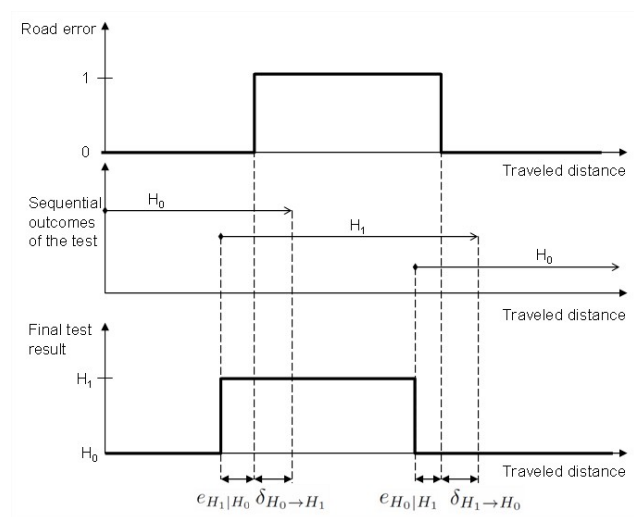

Fig. 3. Definition of the metrics used for tests assessment based on a simple example. The error to detect is represented on the upper part of the figure. As the test may go back in the past to localise a detected error, the outcomes of the test appear on the second line. The error finally detected and localised appears on the bottom graph.

$$
\left\{\begin{array}{c}
H_{0}: d_{i}=b_{i}, i=1, \ldots, N \\
H_{1}: d_{i}=\delta_{m}+b_{i}, i=1, \ldots, N
\end{array}\right.
$$

where $b_{i} \sim \mathcal{N}\left(0, \sigma^{2}\right)$.

On the one hand, a simple decision rule based on the empirical mean of the sliding window was implemented:

$$
\frac{1}{N} \sum_{i=1}^{n} d_{i} \underset{H_{0}}{\stackrel{H_{1}}{\gtrless}} \delta_{m}
$$

On the other hand, the Neyman Pearson probabilistic decision rule was used. This is based on the generalised likelihood ratio of the hypotheses. Under Gaussian noise assumption, the choice follows the rule (29) [15], [18]:

$$
\sum_{i=1}^{n} d_{i} \underset{H_{0}}{\stackrel{H_{1}}{\gtrless}} \sigma \sqrt{2 \cdot N \cdot \log (\Phi)}
$$

Where $\Phi$ arises from a compromise between desired false alarm (type I error) and miss-detection probabilities (type II error) of the decision rule. The false alarm probability has been set to its usual value $(0.1 \%)$ for an appropriate comparison with the proposed method.

The size $N$ of the sliding window must be large enough to be statistically representative and short enough to detect map errors as fast as possible. Moreover, the samples $D$ must be reinitialised as soon as the vehicle leaves one road for another which happens frequently in urban environment. It has then been set to $N=20$ which is equivalent to 250 meters of travelled distance.

\section{Test areas}

The tests were run on a set of roads that was recently modified due to the construction of a new motorway in Normandie, France (see Fig. 4). This area is representative of typical geometric errors that a map may hold and which may cause sever malfunctions in driving assistance systems. In area 1 and 2, sharp bends were added to the road which was previously straight. This would makes a curve

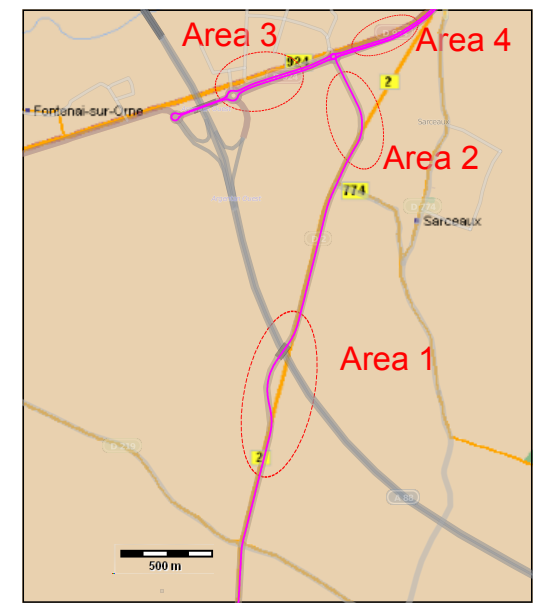

Fig. 4. Global top view of the test areas. The out-of-date map being assessed is shown by yellow lines. A map of the actual road network is plotted in transparent grey. The four test zones are circled in red. The trajectory followed by the vehicle is in purple.

warning system inefficient. In area 3 a new carriage way was added to the old single track road. This induces a constant lateral offset of the new road which would make obsolete intersection warning systems on crossing roads. On the fourth area, the lateral road offset decreases while the vehicle goes. This situation is very useful to highlight the distance to recovery of the tests.

\section{Results analysis}

Tests have been run on the lateral and longitudinal residuals. However, we saw that the most relevant error to detect is lateral road offset. Longitudinal error usually arises from the map-matching filtering process and not from the map itself. We focus therefore solely on the lateral euclidean distance $d$. The most restrictive constraint comes from intersection warning systems which require a longitudinal precision of 10 meters for the placement of an intersection on a road link. Indeed, a lateral offset of a road link induces a longitudinal misplacement of intersection on crossing roads. This value has then been chosen as the mean change to detect $\delta_{m}=10 \mathrm{~m}$. Finally, the cumulative sum threshold is set dynamically to be $\gamma=4 . \sigma / \delta_{m}$ which represents a good compromise between false alarm rate and time to alert.

Results summarized in Table I show that Page's trend test is appropriate for detection and localisation of error in maps. Indeed, errors are detected less than 20 meters after the beginning of the error and well located. The two other methods provide less suitable false alarm and misdetection rates. This is mainly due to the fact that fixed length sliding windows are used. The error detection has then undesirable collateral effects on the tail of the window. These methods show bad results when the road error is small with respect to the size of the sliding window.

Let us focus on area 1, to better understand the strength and weakness of the three methods. The upper part of Fig. 5 shows the lateral error between the vehicle's estimated position and its map-matched position against the travelled 
TABLE I

COMPARATIVE RESULTS WITH NOMINAL TUNING OF EACH TEST

\begin{tabular}{|c|c|c|c|c|c|}
\hline & Test & $\bar{\delta}_{\mathrm{H}_{0} \rightarrow \mathrm{H}_{1}}$ & $\bar{\delta}_{\mathrm{H}_{1} \rightarrow \mathrm{H}_{0}}$ & $\mathrm{e}_{\mathrm{H}_{0}} \mid \mathrm{H}_{1}$ & $\mathrm{e}_{\mathrm{H}_{1} \mid \mathrm{H}_{0}}$ \\
\hline \multirow{3}{*}{ Area 1} & Page & $0 \mathrm{~m}$ & $0 \mathrm{~m}$ & $0 \mathrm{~m}$ & $0 \mathrm{~m}$ \\
\hline & Mean & $73 m$ & $150 \mathrm{~m}$ & $90 \mathrm{~m}$ & $177 \mathrm{~m}$ \\
\hline & N.P. & $46 m$ & $255 \mathrm{~m}$ & $220 \mathrm{~m}$ & $0 \mathrm{~m}$ \\
\hline \multirow{3}{*}{ Area 2} & Page & $20 \mathrm{~m}$ & n.a. ${ }^{3}$ & $20 \mathrm{~m}$ & $0 \mathrm{~m}$ \\
\hline & Mean & n.d. ${ }^{4}$ & n.a. & $35 \mathrm{~m}$ & $0 \mathrm{~m}$ \\
\hline & N.P. & $80 \mathrm{~m}$ & n.a. & $0 \mathrm{~m}$ & $170 \mathrm{~m}$ \\
\hline \multirow{3}{*}{ Area 3} & Page & $0 \mathrm{~m}$ & $0 \mathrm{~m}$ & $0 \mathrm{~m}$ & $0 \mathrm{~m}$ \\
\hline & Mean & $50 m$ & $100 \mathrm{~m}$ & $150 \mathrm{~m}$ & $200 \mathrm{~m}$ \\
\hline & N.P. & $0 \mathrm{~m}$ & $300 \mathrm{~m}$ & $0 \mathrm{~m}$ & $300 \mathrm{~m}$ \\
\hline \multirow{3}{*}{ Area 4} & Page & $0 \mathrm{~m}$ & $20 m$ & $0 \mathrm{~m}$ & $20 m$ \\
\hline & Mean & $50 \mathrm{~m}$ & $0 \mathrm{~m}$ & $250 \mathrm{~m}$ & $40 \mathrm{~m}$ \\
\hline & N.P. & $0 \mathrm{~m}$ & $250 \mathrm{~m}$ & $0 \mathrm{~m}$ & $210 \mathrm{~m}$ \\
\hline
\end{tabular}

distance. The lower part shows the sequential outcomes of each methods while the vehicle is driven. It can be seen on this figure that Page's test is very efficient for detecting the error since it chooses $H_{1}$ as soon as the road is actually erroneous. Moreover, in this example, it locates perfectly the error (from abscissa 520 to 1520 meters). The outcomes of the two other tests are less accurate. Indeed, the errors are detected later and locate it very poorly. This is due to the fact that is not possible to know where the change happened within the sliding window. The whole window is supposed to belong to $H_{1}$ as soon as the threshold is crossed. Correct road points are then declared faulty while they are not and vice versa. This example illustrates why these methods induce false alarms, misdetections and inaccuracies in error localisation.

\section{CONCLUSIONS}

The application of a sequential test on embedded data to detect and localise map errors has been presented. The monitoring of the map consisted in comparing the vehicle map-matched position provided by the navigation engine to the estimated vehicle's position. This estimation was computed by fusing data from the proprioceptive sensors and GNSS estimates. The generated residuals were then analysed using Page's trend test. The presence of a map error was detected when a change in the mean of the observed residuals occurred. This test was run on data collected on real traffic condition and using for reference a three-year-old map, which stored known errors. Trials have shown that every error in the map was rapidly detected and localised accurately (less than $20 \mathrm{~m}$ from the detection distance). The false alarm and misdetection rates were low (no false alarm and less than $20 \mathrm{~m}$ of misdetection over $2500 \mathrm{~m}$ of relevant road sections). The test has shown better performance than conventional algorithms based on fixed length sliding windows. Future work will consist on the implementation of the proposed approach as part of a driving assistance function on a test vehicle to evaluate the benefits of map integrity.

\section{REFERENCES}

[1] "European new car safety programme, speed limitation tests," available at http://www.euroncap.com/Content-Web-Page/7b5a942e-a578-41088c55-2e2dc1d1bceb/safety-assist.aspx, accessed 22 March 2012.

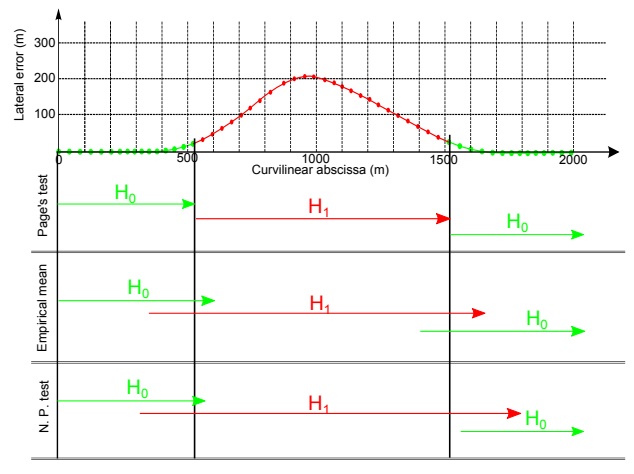

Fig. 5. Sequential outcomes of three trend tests on hypotheses $H_{0}$ (correct road segment) and $H_{1}$ (erroneous road segment). The lateral distance to the map-matched position is denoted on the upper part. The plot colour shows the true state of the road: correct in green, with error in red.

[2] J. Levinson, J. Askeland, J. Becker, J. Dolson, D. Held, S. Kammel, J. Kolter, D. Langer, O. Pink, V. Pratt, M. Sokolsky, G. Stanek, D. Stavens, A. Teichman, M. Werling, and S. Thrun, "Towards fully autonomous driving: Systems and algorithms," in Intelligent Vehicles Symposium (IV), IEEE Baden-Baden, june 2011, pp. 163-168.

[3] I. Jebari, S. Bazeille, E. Battesti, H. Tekaya, M. Klein, A. Tapus, D. Filliat, C. Meyer, Sio-Hoi, Ieng, R. Benosman, E. Cizeron, J.C. Mamanna, and B. Pothier, "Multi-sensor semantic mapping and exploration of indoor environments," in Technologies for Practical Robot Applications (TePRA), IEEE, april 2011, pp. $151-156$.

[4] M. F. Goodchild, "Twenty years of progress: Giscience in 2010," Journal Of Spatial Information Science, vol. 1, pp. 3-20, 2010.

[5] J.-M. Morvan, "Distance and projection," in Generalized Curvatures, ser. Geometry and Computing. Springer Berlin Heidelberg, 2008, vol. 2, pp. 47-56.

[6] T. Badard and C. Lemarie, "Associer des données, 1 appariement," in Généralisation et représentation multiple, 2002.

[7] R. McMaster, "A statistical analysis of mathematical measures for linear simplification," The American Cartographer, vol. 23, 1986.

[8] G. Grekousis and Y. Fotis, "A fuzzy index for detecting spatiotemporal outliers," GeoInformatica, vol. 16, pp. 597-619, 2012.

[9] O. Pink and C. Stiller, "Automated map generation from aerial images for precise vehicle localization," in Intelligent Transportation Systems, ITSC 2010, sept. 2010, pp. $1517-1522$.

[10] T. R. M. Freitas, A. Coelho, and R. Rossetti, "Improving digital maps through gps data processing," in Intelligent Transportation Systems Conference, ITSC 2009, oct. 2009, pp. 1-6.

[11] L. Deren, S. Haigang, and X. Ping, "Automatic change detection of geo-spatial data from imagery," Geo-Spatial Information Science, vol. 6, pp. 1-7, 2003.

[12] S. Schroedl, K. Wagstaff, S. Rogers, P. Langley, and C. Wilson, "Mining gps traces for map refinement," Data Mining and Knowledge Discovery, vol. 9, pp. 59-87, 2004.

[13] G. Agamennoni, J. Nieto, and E. Nebot, "Robust and accurate road map inference," in Robotics and Automation (ICRA), may 2010, pp. $3946-3953$.

[14] C. Hasberg, S. Hensel, and C. Stiller, "Simultaneous localization and mapping for path-constrained motion," Intelligent Transportation Systems, IEEE Transactions on, vol. PP, no. 99, pp. 1 -12, 2011.

[15] M. Basseville and I. V. Nikiforov, Detection of Abrupt Changes: Theory and Application, E. Cliffs, Ed. Prentice-Hall, Inc, 1993.

[16] P. Sundvall and P. Jensfelt, "Fault detection for mobile robots using redundant positioning systems," in International Conference on Robotics and Automation, ICRA, may 2006, pp. $3781-3786$.

[17] P. Bonnifait, P. Bouron, P. Crubille, and D. Meizel, "Data fusion of four abs sensors and gps for an enhanced localization of car-like vehicles," in IEEE International Conference on Robotics and Automation, ICRA, vol. 2, 2001, pp. 1597-1602 vol.2.

[18] D. Maquin and J. Ragot, Diagnostic des systèmes linéaires, Germes, Ed. Lavoisier, 2000.

[19] (2012, March) Ixsea inertial navigation systems homepage. [Online]. Available: http://www.ixsea.com/ 